Original Research Paper

\title{
Social Awareness and Knowledge of Parenteral Viral Hepatitis (B and C) Among Residences of Menoufia Governorate, Egypt: A Questionnaire-Based Field Study
}

\author{
${ }^{1}$ Wesam S Morad, ${ }^{2}$ Maha Elsabaawy and ${ }^{2}$ Mahmoud H Allam \\ ${ }^{1}$ Department of Epidemiology and Preventive Medicine, National Liver Institute, Menoufia University, Egypt \\ ${ }^{2}$ Department of Hepatology and Gastroenterology, National Liver Institute, Menoufia University, Egypt
}

\author{
Article history \\ Received: 21-02-2020 \\ Revised: 29-04-2020 \\ Accepted: 08-05-2020 \\ Corresponding Author: \\ Wesam S. Morad \\ Department of Epidemiology \\ and Preventive Medicine, \\ National Liver Institute, \\ Menoufia University, Egypt \\ Email: wesammorad@gmail.com
}

\begin{abstract}
Egypt had led a unique successful campaign in treating and surveillance of the most prevalent viral infections. However, social awareness evaluation is an unmet need for viral eradication strategic plans. Assess the level of knowledge and awareness of the community about HCV and HBV infections. This community-based cross-sectional survey, was conducted between November 2018 and March 2019 in Menofia Governorate, Egypt. A well-structured pretested questionnaire testing knowledge and awareness regarding $\mathrm{HBV}$ and $\mathrm{HCV}$ infections and their modes of transmission in 14000 medical and non-medical, urban and rural participants. Knowledge about HBV found to be good regarding transmission (81.9\% correct answers), while in cure $51.7 \%$ of participants gave false answers. For HCV infection, good knowledge $(79.3 \%$ of correct answers) was documented, while the curable nature of disease was denied in $40.9 \%$. Blood and blood products $(53.2 \%)$, sexual contact $(27.8 \%)$, mother to child during delivery $(7.3 \%)$ and others were reported as the commonest modes of transmission of $\mathrm{HCV}$ respectively. Television and newspapers were the main sources of knowledge (33 and 22\% respectively). On asking participants about precautions against $\mathrm{HCV}$ infections, $30.2 \%$ stated that they are being educated on this issue, $22.3 \%$ had heard something like that and $47.5 \%$ of participants did not know anything about that. Multivariate logistic regression revealed that for both $\mathrm{HBV}$ and HCV knowledge and awareness were affected by age, residence and level of education. Despite the good results, levels of social awareness should be more elevated for proper viral eradication programs.
\end{abstract}

Keywords: Social Awareness, Knowledge, HCV, HBV, Questionnaire, Field Study

\section{Introduction}

Viral hepatitis is estimated to be the 7th leading cause of mortality worldwide (Stanaway et al., 2016). Hepatitis C Virus (HCV), is a primary cause for liver fibrosis, cirrhosis and cancer which is responsible for one half of this mortality (Mohd Hanafiah et al., 2013; Lavanchy, 2011).

On the other hand, more than 240 million are chronically infected with Hepatitis B Virus (HBV) which is responsible for about 500,000 to 700,000 annual deaths (Toy et al., 2011; WHOEB, 2009; WHO, 2012).
Egypt has the highest prevalence of HCV infection. In which $\mathrm{HCV}$ antibodies sero-prevalence among adult population aged $15-59$ years was $14.7 \%$ in 2009 and at $10.0 \%$ in 2015 which was substantially higher than global levels as stated in The Egypt Demographic and Health Surveys (EDHS) (Mohd Hanafiah et al., 2013; Lavanchy, 2011).

Despite of the lower prevalence of HBV in Egypt (Ismail et al., 2017), it still constitutes the second most common viral infection of the liver which needs effective measures for control. 
To cope with this challenge, Egypt developed a national project for HCV elimination (Gamal, 2014; EESJU, 2014; EMHP, 2017). The National Committee for Control of Viral Hepatitis (NCCVH) was launched in 2006 by the Egyptian Ministry of Health and Population $(\mathrm{MOH})$ to cope with the serious problem of HCV epidemic in the country (El-Akel et al., 2017). On the beginning of its work, The Egyptian NCCVH issued the national treatment strategy for control of $\mathrm{HCV}$ infection, which represented the road map for its work (Doss et al., 2008).

After successful negotiations for $99 \%$ discounted Directly Acting Antiviral drugs (DAA) prices (Kim et al., 2015), Egypt started an ambitious national HCV treatment program with the goal to treat over 250,000 individuals with $\mathrm{HCV}$ infection per year, with the hope of reduction of $\mathrm{HCV}$ prevalence to $<2 \%$ by 2025 (McNeil Jr., 2015).

Several studies suggest that the incidence of HCV infection has decreased since the second half of the 20th century. First, most countries have age-specific prevalence of serological evidence of past or present infection, suggesting lower incidence in recent years (Bruggmann et al., 2014; Saraswat et al., 2015; Liakina et al., 2015; Armstrong et al., 2000). Second, countries that conduct surveillance for acute hepatitis $\mathrm{C}$ reported decreases in the rates (Williams et al., 2011). Third, countries that conducted more than one biomarker survey, such as Egypt, reported an evolution over time that suggests a decrease in incidence (MHPICFI, 2015). Fourth, injection safety improved, which reduced the incidence of injection-associated HCV infection (Pépin et al., 2014).

Worldwide, $7 \%$ of those diagnosed (1.1 million) were started on treatment in 2015. The Eastern Mediterranean Region accounted for the largest proportion of those started on treatment (12\%), boosted by the large-scale elimination plans in Egypt (Estes et al., 2015). Of those started on treatment in 2015, about half received DAAs. Given that more people were initiated on treatment the following year, WHO (2016) global report on access to hepatitis $\mathrm{C}$ treatment estimated that about 1 million persons had accessed DAAs in selected countries. However, there is wide variation in terms of access to DAAs from country to country.

For example, in 2015, the HCV elimination program in Egypt was based on the use of DAAs.

These measures could only succeed if based on good knowledge and awareness of both infections by community members who require comprehensive contribution of both health care delivery system and the Egyptian community.

Thus, this study was conducted on a cohort of population in order to assess the level of knowledge and awareness of the community about $\mathrm{HCV}$ and HBV infections.

\section{Study Design and Data Collection}

This community based cross sectional survey was conducted between November 2018 and March 2019 in Menofia Governorate which is located at the Nile delta at north of Egypt. This Governorate is populous with a surface area of $2,543 \mathrm{~km}^{2}$ and a population number of 4,077 million.

We excluded those $<18$ years old and those who refused to participate in the study from the start or who refused to complete the questionnaire.

The study was done by using a well-structured pretested questionnaire containing 10 closed-ended (yes/no) and 8 open-ended questions. The questionnaire tested the demographic and socioeconomic characteristics of participants along with 19 questions testing knowledge and awareness regarding $\mathrm{HBV}$ and $\mathrm{HCV}$ infections and their modes of transmission.

The awareness about prevention of HBV and HCV infections including vaccination status was included in a set of questions with a focus on the source of participants' information. Also, the screening status of participants and their family members was included in these questions, Tables 3, 4, 7 and 8 .

This questionnaire was developed on basis of previous studies (Du et al., 2012; Denniston et al., 2012). The original questionnaires were in English and some of its questions were not suitable with the Egyptian culture. So, we chose some of these questions and translated it to simple questions that could be easily understood. The questionnaire was revised and modified several times by some professors of National liver institute. A small pilot study was done on 50 of the employees of the National Liver Institute and some of the companions of the attending patients to the outpatient clinic of the National Liver Institute hospital before it was finally approved.

The study was done with the help of 5 interviewers (including 4 nurses and one employee from National Liver Institute). The idea of the study and items of the questionnaire was explained to all of the interviewers before starting the study.

Printed copies of the questionnaire were distributed to 14682 participants using systematic random sampling technique by the interviewers.

Of the 14,682 participants, 14000 were recruited in which 682 individuals refused to participate in the study; 1000 from medical students of the Faculty of Medicine, Menoufia University, 3000 were non-medical students from three faculties other than the faculty of medicine belonging to the same University. Five thousand residents were recruited from five rural areas of Menofia Governorate and lastly 5000 residents were chosen from other five urban centers of the same Governorate.

After obtaining verbal approval to be included in the study, all participants were asked to fill the 
administered questionnaire at their own will and convenience. Then filled questionnaires were anonymously returned to the interviewers. The interviewers helped some participants who felt difficulty to understand some questions and they filled the questionnaire by themselves for illiterate persons.

\section{Important Definitions}

However, there is a distinct difference between awareness and knowledge.

Awareness is perceiving, knowing, feeling, or being conscious of events, objects, thoughts, emotions, or sensory patterns.

Knowledge is facts, information and skills acquired through experience or education.

A knowledge score depending on the mean percentage of correct answers was assigned. A percentage of correct answers equal to or greater than $60 \%$ of all questions was considered "good", if less than $60 \%$ or equal poor.

Statistical Analysis Data were coded, tabulated and analyzed using the Statistical Package for Social Science (SPSS) version 26.0 for Windows (SPSS, Chicago, IL, USA). Continuous variables were described using mean and standard deviation and categorical variables were described using frequencies and percentages. The $\mathrm{z}$ ratio was used for the significance of the difference between two independent proportions and a $p$ value less than 0.05 was considered statistically significant. The Analysis of Variance (ANOVA) test was used for the significance of the difference between quantitative variables and a $p$-value less than 0.05 was considered statistically significant.

\section{Results}

\section{Socio-Demographics of Participants}

A sum of 14682 inhabitants of Menofia Governorate voluntarily participated in this study. Participants were asked to fill out the study questionnaire under supervision of the interviewers. Among all participants, 682 individuals returned unfilled questionnaires or refused to continue the interview (response rate, 95.4\%) and they were excluded from the study.
Of all participants, males were 7630 (54.5\%) while females were $6370(45.5 \%)$ with mean age ranging from $42 \pm 12$ years.

As regard students' group (medical and non-medical), $(55 \%)$ were males and $(45 \%)$ were females with mean age $24.6 \pm 1.1$ years ranging between $22-28$ yrs.

While for public residents group (urban and rural), males were $(47.4 \%$ and $47.7 \%)$ and females were $(52.6 \%$ and $52.3 \%)$ with mean age $(46.4 \pm 16.2$ and $47.2 \pm 17.4$ years) and range between (18-65 and 20- 67 years) for residents of rural and urban areas respectively.

According to the socioeconomic level of these residents, $(48.7 \%$ and $49.4 \%)$ were of low and intermediate levels and $(51.3 \%$ and $50.6 \%)$ of high socioeconomic levels for residents of rural and urban areas respectively.

About $49.3 \%$ and $56.2 \%$ of participants underwent previous screening for hepatitis $\mathrm{B}$ and $\mathrm{C}$ respectively while $5.6 \%$ and $7.2 \%$ were not sure about $\mathrm{HBV}$ and HCV screening respectively.

\section{Knowledge About HBV}

Knowledge about HBV was tested by questions 1 to 6 , Table 1. Answers of participants revealed good knowledge $(81.9 \%$ correct answers) regarding $\mathrm{HBV}$ transmission, Table 1 and Fig. 1. But, $51.7 \%$ of participants gave false answers about HBV cure, Table 1.

There was no significant difference between percentage of correct answers between medical and nonmedical students and between residents of rural or urban areas, Table 2.

Knowledge of participants about HBV transmission was significantly affected by their age category, residence area, current jobs, level of education and socioeconomic standard measured by monthly income $(\mathrm{p}<0.05)$, Table 3 .

While gender of participants and their marital status did not affect their knowledge about HBV infection, Table 3.

As regard modes of $\mathrm{HBV}$ transmission, blood and blood products transfusions $(50.9 \%)$, followed by sexual contact $(30.1 \%)$ and from mother to child during delivery $(6.7 \%)$ were reported as the common modes of HBV infection, Fig. 1.

Table 1: Correct answers to questions testing knowledge and awareness about HBV infection among all participants.

\begin{tabular}{lll}
\hline Statements & & Correct answers N (\%) \\
\hline Knowledge & Is hepatitis B an infectious/transmissible disease? & $12614(90.1)$ \\
& What is the causative agent for hepatitis B? & $13342(95.3)$ \\
& Which organ is more affected by Hepatitis B? & $13608(97.2)$ \\
& Should every patient undergoing surgery be screened for HBV? & $10234(73.1)$ \\
& Is screening of blood donors for HBV mandatory for safe transfusion? & $12516(89.4)$ \\
& Can hepatitis B patient be cured completely by treatment? & $6902(49.3)$ \\
Awareness & Is hepatitis B infection a preventable disease? & $12852(91.8)$ \\
& What are measures to prevent hepatitis B? & $13034(93.1)$ \\
& Is there any available vaccine for hepatitis B? & $13636(97.4)$ \\
& What are the minimum needed doses of the vaccine to complete vaccination against HBV? & $12068(86.2)$ \\
\hline
\end{tabular}


Table 2: Comparison of correct answers about knowledge and awareness about HBV infection among different groups of participants

\begin{tabular}{|c|c|c|c|c|c|c|c|c|}
\hline \multirow[b]{3}{*}{ Statements } & \multicolumn{4}{|c|}{ Correct responses $(\%)$} & \multirow{2}{*}{\multicolumn{4}{|c|}{$\mathrm{p}$ value }} \\
\hline & \multirow{2}{*}{$\begin{array}{l}\text { Non-medical } \\
\text { students }(3000)\end{array}$} & \multirow{2}{*}{$\begin{array}{l}\text { Medical } \\
\text { students (1000) }\end{array}$} & \multirow{2}{*}{$\begin{array}{l}\text { Residents at rural } \\
\text { areas }(5000)\end{array}$} & \multirow{2}{*}{$\begin{array}{l}\text { Residents at urban } \\
\text { areas }(5000)\end{array}$} & & & & \\
\hline & & & & & $\mathrm{P} 1$ & $\mathrm{P} 2$ & P3 & $\mathrm{P} 4$ \\
\hline Question 1 & 87.2 & 95.2 & 74.3 & 90.8 & 0.042 & 0.044 & 0.40 & 0.82 \\
\hline Question 2 & 89.5 & 98.0 & 92.4 & 93.0 & 0.005 & 0.81 & & \\
\hline Question 3 & 96.1 & 98.8 & 97.0 & 93.6 & 0.38 & 0.12 & & \\
\hline Question 4 & 65.7 & 64.5 & 82.0 & 72.9 & 0.75 & 0.26 & & \\
\hline Question 5 & 79.0 & 90.4 & 94.0 & 93.9 & 0.03 & 0.84 & & \\
\hline Question 6 & 42.4 & 41.5 & 36.6 & 39.0 & 0.76 & 0.90 & & \\
\hline Question 7 & 84.2 & 92.2 & 84.0 & 97.9 & 0.12 & 0.043 & & \\
\hline Question 8 & 87.0 & 93.0 & 87.3 & 93.4 & 0.09 & 0.48 & 0.31 & 1.01 \\
\hline Question 9 & 95.0 & 98.6 & 97.2 & 96.0 & 0.23 & 0.71 & & \\
\hline Question 10 & 84.0 & 87.2 & 81.8 & 67.9 & 0.47 & 0.15 & & \\
\hline
\end{tabular}

p- value < 0.05 , considered statistical significant; $\mathrm{p}$-value by $\mathrm{Z}$ ratio; $\mathrm{p} 1$ and $\mathrm{p} 3=$ non-medical vs. medical students; $\mathrm{p} 2$ and $\mathrm{p} 4=$ residents of rural vs. urban areas

Table 3: knowledge of participants about HBV prevention, $(\mathrm{n}=14000)$.

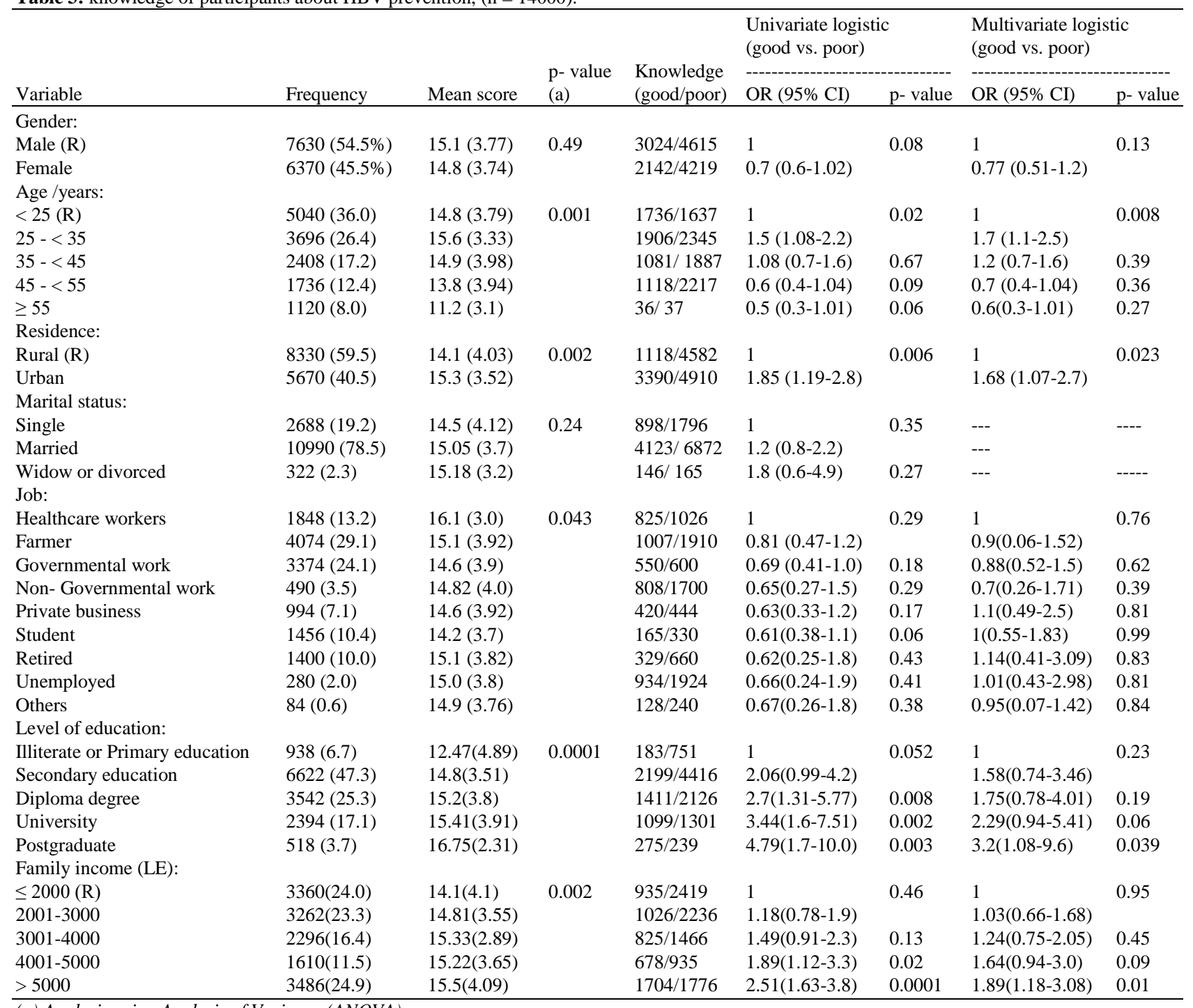

(a) Analysis using Analysis of Variance (ANOVA)

CI confidence interval, OR odds ratio, $R$ reference group, LE Egyptian pounds, SD standard deviation 


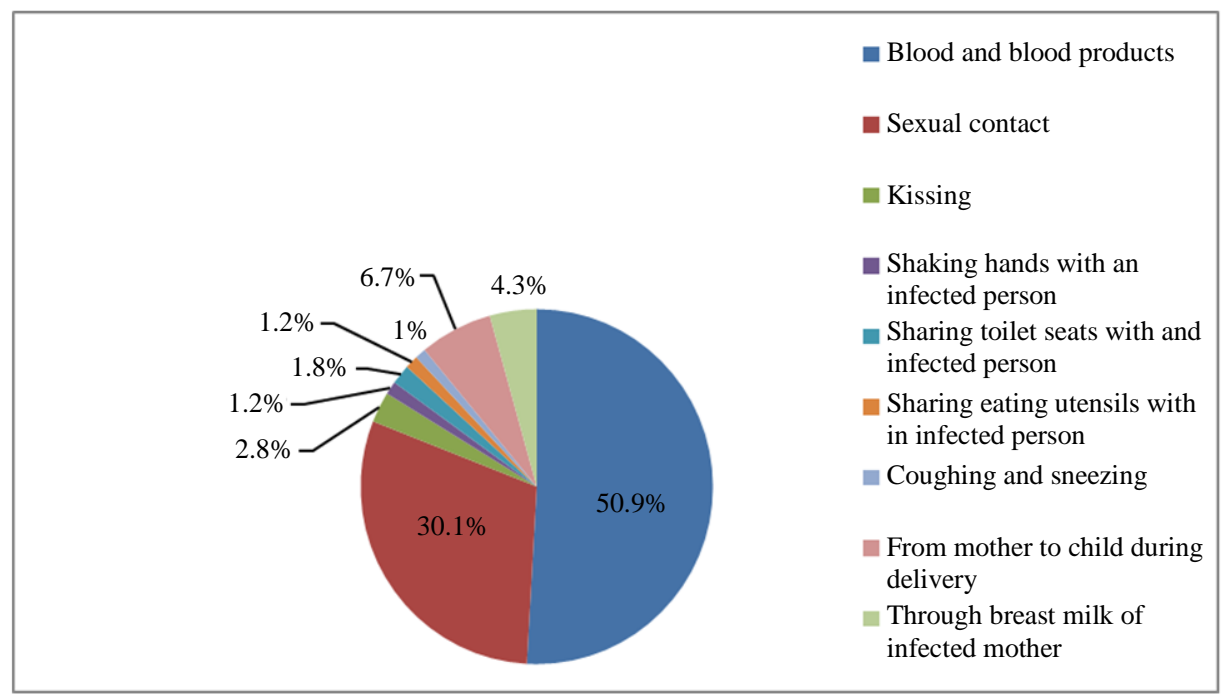

Fig. 1: Knowledge of modes of HBV transmission among 14000 person allover Menoufia Governorate in Egypt

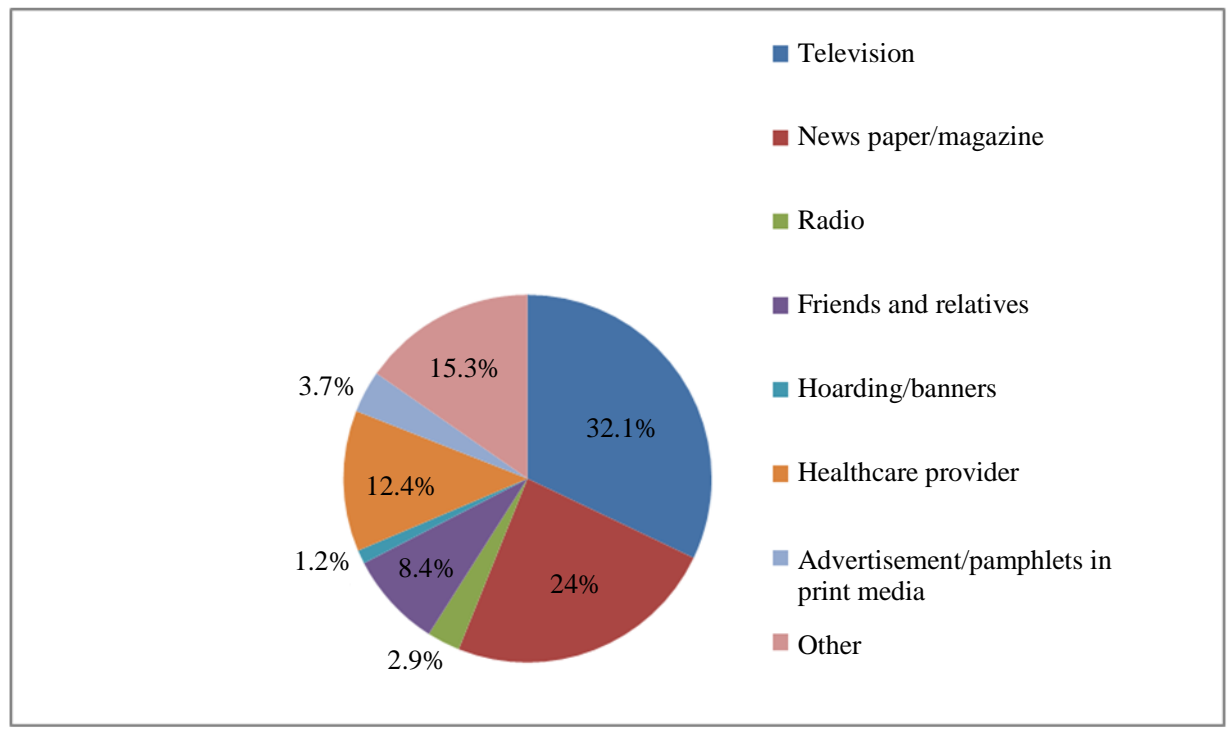

Fig. 2: Source of knowledge about hepatitis B among 14000 person allover Menoufia Governorate in Egypt

About $72.6 \%$ of participants were vaccinated for hepatitis B infection while $7.2 \%$ did not know about their vaccination status. Amazingly, $47.3 \%$ participants stated that none of their family members were vaccinated against HBV and $21.5 \%$ were not sure whether about vaccination status of their family members.

Source of participants' knowledge was variable. About $(32.1 \%)$ gained their knowledge from television materials and $(24 \%)$ of them gained it from newspapers and magazines, Fig. 2.

\section{Awareness About HBV}

Awareness about HBV preventive measures was tested by questions number 7 to 10 , Table 1 . Participants had good awareness about measures of HBV prevention and availability of HBV vaccine with $(92.13 \%)$ correct answers to supplied questions.

Percentage of correct answers was not significantly different among different study groups, Table 2 .

On Univariate analysis, age category, residence, marital status and level of education of participants was significantly related to their level of awareness about HBV prevention. Whereas, gender, job and no monthly income had no significant relationship, Table 4.

\section{Knowledge About HCV}

The study revealed good knowledge $(79.3 \%$ of correct answers) regarding $\mathrm{HCV}$ infection (reflected by the first six questions in Table 5) among all participants except for the curable nature of $\mathrm{HCV}$, 
where $40.9 \%$, of participants gave an answer denying that patients can be completely cured of HCV infection, Table 5 and Fig. 3.

The percent of correct answers did not differ between medical and non-medical students and between residents of rural and urban areas, Table 6 .
Knowledge about HCV transmission was significantly related to participants' age category, residence area, current jobs and level of education and socioeconomic standard of participants. While, gender and marital status of participants had no significant relationship, Table 7 .

Table 4: Awareness of participants about HBV infection prevention, $(n=14000)$

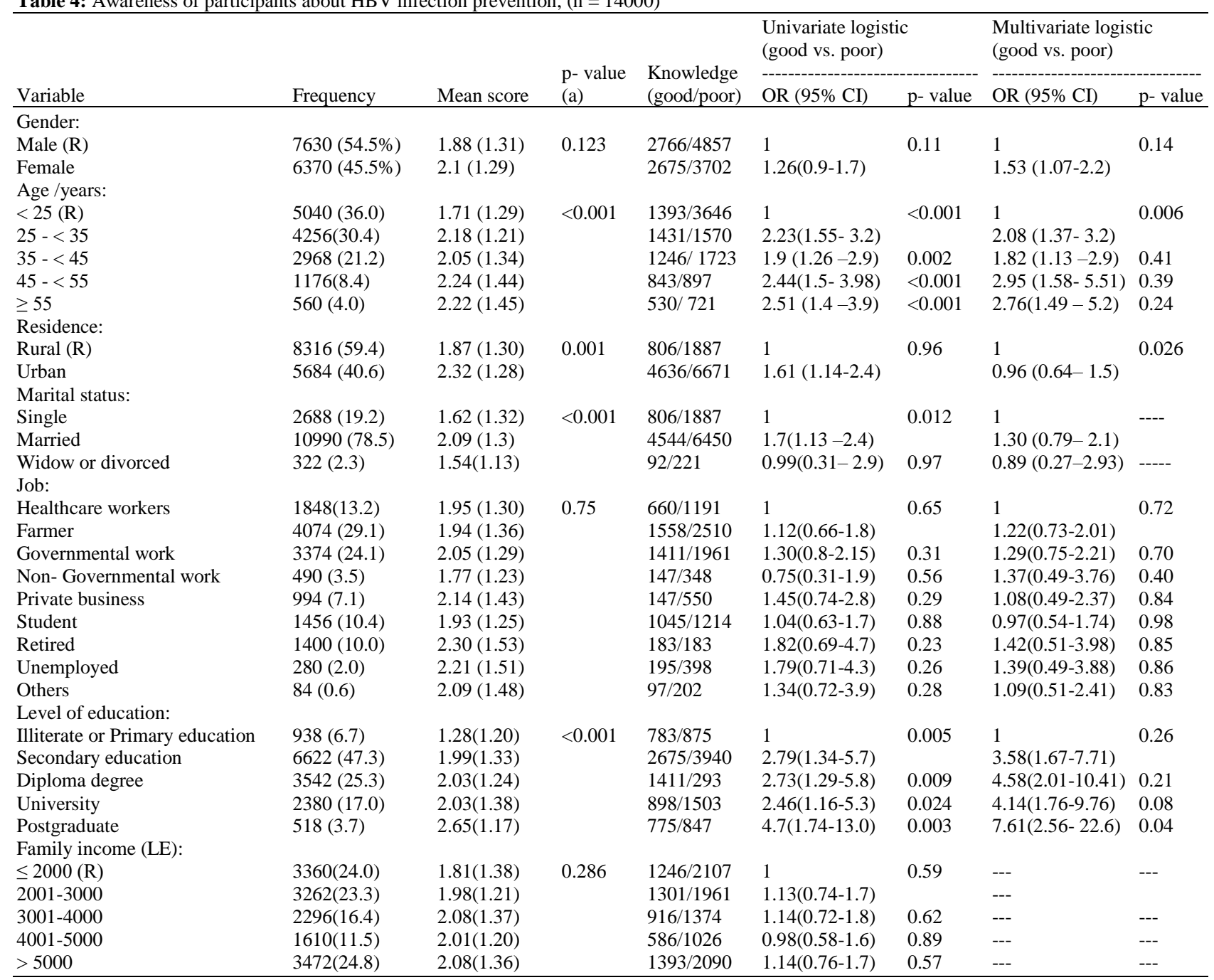

(a) Analysis using Analysis of Variance (ANOVA)

CI confidence interval, OR odds ratio, R reference group, LE Egyptian pounds, SD standard deviation.

Table 5: Correct answers to questions testing knowledge and awareness about HCV infection among all participants.

\begin{tabular}{llc}
\hline Statements & & Correct answers N (\%) \\
\hline Knowledge & Is hepatitis C an infectious/transmissible disease? & $12474(89.1)$ \\
& What is the causative agent for hepatitis C? & $13104(93.6)$ \\
& Which organ is more affected by Hepatitis C? & $13552(96.8)$ \\
& Should every patient undergoing surgery be screened for HCV? & $9576(68.4)$ \\
& Is screening of blood donors for HCV mandatory for safe transfusion? & $12208(87.2)$ \\
Awareness & Can hepatitis C patient be cured completely by treatment? & $5726(40.9)$ \\
& Is hepatitis C infection a preventable disease? & $12474(89.1)$ \\
& What are measures to prevent hepatitis C? & $12614(90.1)$ \\
& Is there any available vaccine for hepatitis C? & $13552(96.8)$ \\
\hline
\end{tabular}


Table 6: Comparison of correct answers about knowledge and awareness about HCV infection among different groups of participants

\begin{tabular}{|c|c|c|c|c|c|c|c|c|}
\hline \multirow[b]{3}{*}{ Statements } & \multicolumn{4}{|c|}{ Correct responses $(\%)$} & \multirow{2}{*}{\multicolumn{4}{|c|}{$\mathrm{p}$ value }} \\
\hline & \multirow{2}{*}{$\begin{array}{l}\text { Non-medical } \\
\text { students }(3000)\end{array}$} & \multirow{2}{*}{$\begin{array}{l}\text { Medical } \\
\text { students (1000) }\end{array}$} & \multirow{2}{*}{$\begin{array}{l}\text { Rural residence } \\
\text { individuals' }\end{array}$} & \multirow{2}{*}{$\begin{array}{l}\text { Urban residence } \\
\text { individuals' }\end{array}$} & & & & \\
\hline & & & & & $\mathrm{P} 1$ & $\mathrm{P} 2$ & $\mathrm{P} 3$ & $\mathrm{P} 4$ \\
\hline Question 1 & 91.9 & 98.7 & 85.7 & 92.1 & 0.04 & 0.041 & 0.39 & 0.80 \\
\hline Question 2 & 81.0 & 97.3 & 92.0 & 93.9 & 0.003 & 0.80 & & \\
\hline Question 3 & 94.7 & 98.0 & 98.1 & 93.0 & 0.36 & 0.10 & & \\
\hline Question 4 & 78.2 & 74.3 & 84.2 & 79.9 & 0.73 & 0.22 & & \\
\hline Question 5 & 80.4 & 90.0 & 94.8 & 93.0 & 0.03 & 0.81 & & \\
\hline Question 6 & 58.3 & 55.3 & 43.2 & 40.2 & 0.72 & 0.89 & & \\
\hline Question 7 & 88.4 & 92.0 & 87.2 & 96.2 & 0.11 & 0.04 & & \\
\hline Question 8 & 89.6 & 94.2 & 88.1 & 89.2 & 0.07 & 0.46 & 0.29 & 1 \\
\hline Question 9 & 94.8 & 98.0 & 95.7 & 96.0 & 0.21 & 0.70 & & \\
\hline
\end{tabular}

$\mathrm{p}<0.05$, considered statistical significant; $\mathrm{p}$ value by $\mathrm{Z}$ ratio; $\mathrm{p} 1$ and $\mathrm{p} 3=$ non-medical vs. medical students; $\mathrm{p} 2$ and $\mathrm{p} 4=$ residents of rural vs. Urban areas

Table 7: knowledge of participants about HCV infection, $(\mathrm{n}=14000)$.

\begin{tabular}{|c|c|c|c|c|c|c|c|c|}
\hline \multirow[b]{2}{*}{ Variable } & \multirow[b]{2}{*}{ Frequency } & \multirow[b]{2}{*}{ Mean score } & \multirow[b]{2}{*}{$\begin{array}{l}\text { p- value } \\
\text { (a) }\end{array}$} & \multirow[b]{2}{*}{$\begin{array}{l}\text { Knowledge } \\
\text { (good/poor) }\end{array}$} & \multicolumn{2}{|c|}{$\begin{array}{l}\text { Univariate logistic } \\
\text { (good vs. poor) }\end{array}$} & \multicolumn{2}{|c|}{$\begin{array}{l}\text { Multivariate logistic } \\
\text { (good vs. poor) }\end{array}$} \\
\hline & & & & & OR $(95 \% \mathrm{CI})$ & p-value & OR $(95 \% \mathrm{CI})$ & $\mathrm{p}$-value \\
\hline \multicolumn{9}{|l|}{ Gender: } \\
\hline Male (R) & $7520(53.7 \%)$ & $14.9(3.74)$ & \multirow[t]{2}{*}{0.46} & $3034 / 4505$ & 1 & \multirow[t]{2}{*}{0.09} & 1 & \multirow[t]{2}{*}{0.14} \\
\hline Female & $6480(46.3 \%)$ & $13.9(3.54)$ & & $2192 / 4269$ & $0.73(0.7-1.1)$ & & $0.82(0.53-1.4)$ & \\
\hline \multicolumn{9}{|l|}{ Age /years: } \\
\hline$<25(\mathrm{R})$ & $4910(35.07)$ & $13.9(3.73)$ & \multirow[t]{5}{*}{0.002} & $1700 / 1643$ & 1 & \multirow[t]{2}{*}{0.03} & 1 & \multirow[t]{2}{*}{0.006} \\
\hline $25-<35$ & $3416(24.4)$ & $15.2(3.31)$ & & $1806 / 2355$ & $1.49(1.1-2.3)$ & & $1.78(1.13-2.3)$ & \\
\hline $35-<45$ & $2508(17.9)$ & $14.5(3.78)$ & & $1181 / 1860$ & $1.1(0.72-1.6)$ & 0.71 & $1.22(0.74-1.7)$ & 0.41 \\
\hline $45-<55$ & 1956 (13.97) & $14.1(3.82)$ & & $1125 / 2237$ & $0.6(0.5-1.14)$ & 0.08 & $0.72(0.44-1.06)$ & 0.39 \\
\hline$\geq 55$ & $1210(8.64)$ & $11.5(3.23)$ & & $43 / 50$ & $0.53(0.4-1.0)$ & 0.07 & $0.65(0.33-1.1)$ & 0.24 \\
\hline \multicolumn{9}{|l|}{ Residence: } \\
\hline Rural (R) & 8105 (57.9) & $14.2(4.13)$ & \multirow[t]{2}{*}{0.003} & $1008 / 4592$ & 1 & \multirow[t]{2}{*}{0.005} & 1 & \multirow[t]{2}{*}{0.026} \\
\hline Urban & $5895(42.1)$ & $15.4(3.49)$ & & $3440 / 4960$ & $1.87(1.2-2.77)$ & & $1.78(1.1-2.75)$ & \\
\hline \multicolumn{9}{|l|}{ Marital status: } \\
\hline Single & $2408(17.2)$ & $14.7(4.32)$ & \multirow[t]{4}{*}{0.26} & $918 / 1726$ & 1 & \multirow[t]{2}{*}{0.37} & --- & \multirow[t]{2}{*}{----} \\
\hline Married & $10790(77.1)$ & $15.21(3.9)$ & & $4143 / 6092$ & $1.3(0.7-2.31)$ & & --- & \\
\hline Widow or divorced & $802(5.7)$ & $15.23(3.3)$ & & $476 / 645$ & $1.7(0.5-4.87)$ & 0.28 & --- & ----- \\
\hline \multicolumn{8}{|l|}{ Job: } & \\
\hline Healthcare workers & $1600(11.4)$ & $16.13(3.2)$ & \multirow[t]{9}{*}{0.045} & 710/1041 & 1 & 0.31 & 1 & 0.72 \\
\hline Farmer & $4274(30.5)$ & $15.3(3.94)$ & & $1207 / 1710$ & $0.83(0.5-1.22)$ & & $0.91(0.1-1.57)$ & \\
\hline Governmental work & $3382(24.2)$ & $14.61(4.0)$ & & $590 / 660$ & $0.71(0.43-1.1)$ & 0.21 & $0.88(0.52-1.62)$ & 0.70 \\
\hline Non- Governmental work & $530(3.8)$ & $14.85(4.1)$ & & $600 / 1808$ & $0.69(0.29-1.6)$ & 0.27 & $0.76(0.36-1.77)$ & 0.40 \\
\hline Private business & $854(6.1)$ & $14.62(3.9)$ & & $470 / 494$ & $0.66(0.35-1.3)$ & 0.19 & $1.13(0.52-2.52)$ & 0.84 \\
\hline Student & $1476(10.5)$ & $14.21(3.8)$ & & $135 / 300$ & $0.64(0.37-1.2)$ & 0.08 & $1.23(0.59-1.87)$ & 0.98 \\
\hline Retired & $1520(10.86)$ & $15.17(3.8)$ & & $339 / 680$ & $0.68(0.23-1.7)$ & 0.40 & $1.16(0.46-3.19)$ & 0.85 \\
\hline Unemployed & $260(1.86)$ & $15.3(3.72)$ & & $940 / 1938$ & $0.69(0.25-1.8)$ & 0.45 & $1.11(0.48-2.94)$ & 0.86 \\
\hline Others & $104(0.74)$ & $15.1(3.79)$ & & $132 / 246$ & $0.66(0.3-1.77)$ & 0.41 & $0.99(0.17-1.52)$ & 0.83 \\
\hline Level of education: & & & & & & & & \\
\hline Illiterate or Primary education & $900(6.4)$ & $12.67(4.9)$ & 0.0002 & $213 / 521$ & 1 & 0.05 & 1 & 0.26 \\
\hline Secondary education & $6640(47.4)$ & $14.84(3.6)$ & & $2099 / 4516$ & $2.56(0.9-4.33)$ & & $1.61(0.71-3.76)$ & \\
\hline Diploma degree & $3562(25.4)$ & $15.21(3.82)$ & & $1461 / 2176$ & 2.74(1.4-5.81) & 0.007 & $1.79(0.88-4.1)$ & 0.21 \\
\hline University & $2234(15.95)$ & $15.51(4.0)$ & & $1149 / 1201$ & $3.46(1.6-7.53)$ & 0.001 & $2.31(0.90-5.46)$ & 0.08 \\
\hline Postgraduate & $664(4.74)$ & $16.76(2.33)$ & & $375 / 289$ & $4.82(1.8-10.1)$ & 0.002 & $3.24(1.18-9.66)$ & 0.04 \\
\hline Family income (LE): & & & & & & & & \\
\hline$\leq 2000(\mathrm{R})$ & $3220(23.0)$ & $14.43(4.21)$ & 0.003 & $825 / 2429$ & 1 & 0.49 & 1 & 0.98 \\
\hline $2001-3000$ & $3402(24.3)$ & $14.89(3.59)$ & & $1056 / 2286$ & $1.16(0.82-1.7)$ & & $1.13(0.86-1.78)$ & \\
\hline $3001-4000$ & $2230(15.9)$ & $15.53(2.92)$ & & $845 / 1336$ & $1.52(0.92-2.1)$ & 0.17 & $1.26(0.79-2.15)$ & 0.47 \\
\hline $4001-5000$ & $1626(11.6)$ & $15.32(3.71)$ & & 778/965 & $1.91(1.22-3.1)$ & 0.04 & $1.72(0.91-3.4)$ & 0.11 \\
\hline$>5000$ & $3522(25.2)$ & $15.58(4.12)$ & & $1604 / 1876$ & $2.53(1.65-3.7)$ & 0.0002 & $1.91(1.38-3.18)$ & 0.02 \\
\hline
\end{tabular}

(a) Analysis using Analysis of Variance (ANOVA).

CI confidence interval, OR odds ratio, R reference group, LE Egyptian pounds, SD standard deviation. 


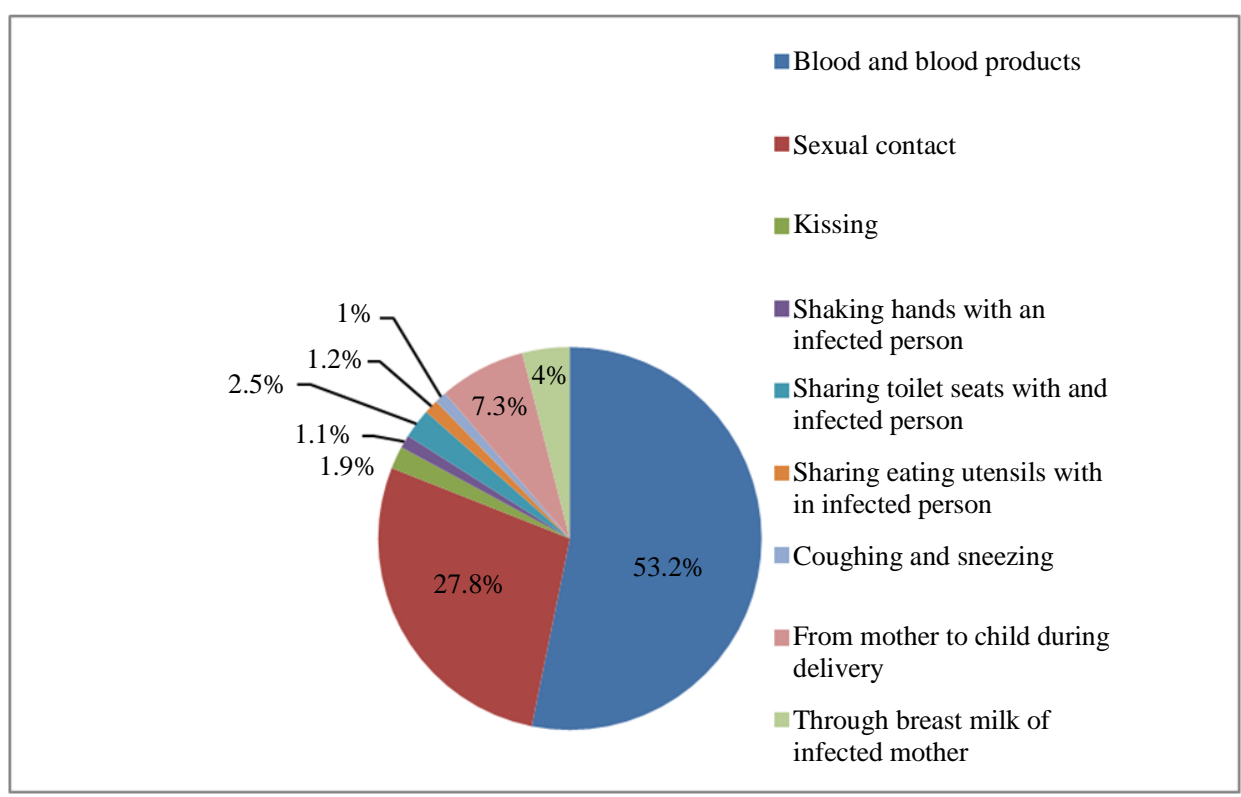

Fig. 3: Knowledge of modes of HCV transmission among 14000 person allover Menoufia Governorate in Egypt

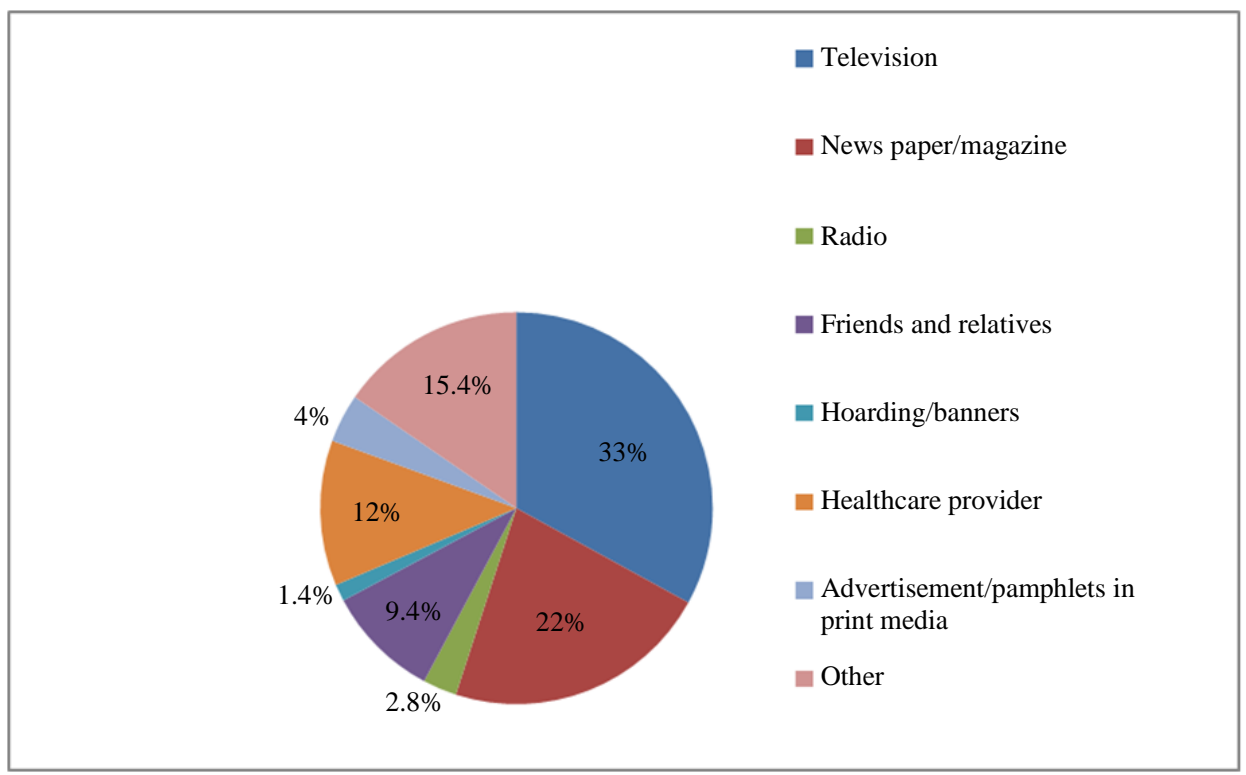

Fig. 4: Source of knowledge about hepatitis C among 14000 person allover Menoufia Governorate in Egypt

Blood and blood products $(53.2 \%)$, sexual contact $(27.8 \%)$, mother to child during delivery $(7.3 \%)$ and others were reported as the commonest modes of transmission of HCV respectively, Fig. 4.

Participants stated that they had their knowledge mainly from television materials and newspapers (33 and $22 \%$ respectively), Fig. 4.

\section{Awareness About HCV}

The awareness of participants about measures to prevent HCV was tested by the last 3 questions in Table
5. About $(92 \%)$ of participants gave correct answers on these questions.

Age of participants, their residence area, marital status and education level varied significantly with their awareness about prevention of $\mathrm{HCV}$ infection, Table 8 .

On asking participants about precautions against $\mathrm{HCV}$ infections, $30.2 \%$ stated that they are being educated on this issue, $22.3 \%$ had heard something like that and $47.5 \%$ of participants did not know anything about that. 
Table 8: Awareness of participants about HCV prevention, $(n=14000)$

\begin{tabular}{|c|c|c|c|c|c|c|c|c|}
\hline \multirow[b]{2}{*}{ Variable } & \multirow[b]{2}{*}{ Frequency } & \multirow[b]{2}{*}{ Mean score } & \multirow[b]{2}{*}{$\begin{array}{l}\text { p-value } \\
\text { (a) }\end{array}$} & \multirow[b]{2}{*}{$\begin{array}{l}\text { Knowledge } \\
\text { (good/poor) }\end{array}$} & \multicolumn{2}{|c|}{$\begin{array}{l}\text { Univariate logistic } \\
\text { (good vs. poor) }\end{array}$} & \multicolumn{2}{|c|}{$\begin{array}{l}\text { Multivariate logistic } \\
\text { (good vs. poor) }\end{array}$} \\
\hline & & & & & OR $(95 \%$ CI $)$ & $\mathrm{p}$ - value & OR $(95 \% \mathrm{CI})$ & p- value \\
\hline \multicolumn{9}{|l|}{ Gender: } \\
\hline Male (R) & $7230(51.6 \%)$ & $1.82(1.39)$ & \multirow[t]{2}{*}{0.126} & $2706 / 4837$ & 1 & \multirow[t]{2}{*}{0.12} & 1 & \multirow[t]{2}{*}{0.14} \\
\hline Female & $6770(48.4 \%)$ & $2.3(1.31)$ & & $2735 / 3722$ & $1.24(1.0-1.71)$ & & $1.57(1.13-2.3)$ & \\
\hline \multicolumn{9}{|l|}{ Age /years: } \\
\hline $25-<35$ & $4276(30.5)$ & $2.22(1.29)$ & \multirow{4}{*}{$<0.001$} & $1541 / 1670$ & $2.21(1.59-3.0)$ & $<0.001$ & $2.11(1.34-3.14)$ & 0.006 \\
\hline $35-<45$ & $2338(16.7)$ & $2.11(1.39)$ & & $1226 / 1503$ & $1.9(1.3-2.87)$ & 0.001 & $1.82(1.13-2.8)$ & 0.41 \\
\hline $45-<55$ & $1286(9.2)$ & $2.27(1.49)$ & & $863 / 998$ & $2.48(1.59-3.9)$ & $<0.001$ & $2.91(1.62-5.54)$ & 0.39 \\
\hline$\geq 55$ & $1080(7.7)$ & $2.20(1.42)$ & & $650 / 640$ & $2.5(1.44-3.8)$ & $<0.001$ & $2.82(1.45-5.3)$ & 0.24 \\
\hline \multicolumn{9}{|l|}{ Residence: } \\
\hline Rural (R) & $8106(57.9)$ & $1.89(1.31)$ & \multirow[t]{2}{*}{0.002} & $800 / 1893$ & 1 & \multirow[t]{2}{*}{0.91} & 1 & \multirow[t]{2}{*}{0.026} \\
\hline Urban & $5894(42.1)$ & $2.34(1.29)$ & & $4536 / 6771$ & $1.67(1.2-2.44)$ & & $0.98(0.6-1.48)$ & \\
\hline \multicolumn{9}{|l|}{ Marital status: } \\
\hline Married & $11140(79.6)$ & $2.12(1.33)$ & \multirow{3}{*}{$<0.001$} & $4624 / 6670$ & $1.73(1.1-2.5)$ & 0.014 & $1.32(0.81-2.2)$ & ---- \\
\hline Widow or divorced & $432(3.1)$ & $1.57(1.19)$ & & $231 / 402$ & $0.97(0.3-2.82)$ & 0.93 & $0.91(0.24-2.98)$ & ----- \\
\hline \multicolumn{8}{|l|}{ Job: } & \\
\hline Healthcare workers & $1408(10.1)$ & $1.98(1.34)$ & \multirow[t]{9}{*}{0.78} & $500 / 1291$ & 1 & 0.71 & 1 & 0.72 \\
\hline Farmer & $4184(29.9)$ & $1.96(1.37)$ & & $1588 / 2530$ & $1.14(0.68-1.7)$ & & $1.24(0.77-2.12)$ & \\
\hline Governmental work & $3474(24.8)$ & $2.08(1.31)$ & & $1011 / 1661$ & $1.3(0.82-2.13)$ & 0.39 & $1.25(0.77-2.23)$ & 0.70 \\
\hline Non- Governmental work & $520(3.7)$ & $1.79(1.29)$ & & $357 / 548$ & $0.79(0.3-1.98)$ & 0.61 & $1.33(0.51-3.81)$ & 0.40 \\
\hline Private business & $964(6.9)$ & $2.22(1.52)$ & & $347 / 450$ & $1.49(0.8-2.79)$ & 0.31 & $1.11(0.49-2.39)$ & 0.84 \\
\hline Student & $1426(10.2)$ & $1.95(1.29)$ & & $1155 / 1004$ & $1.1(0.69-1.78)$ & 0.84 & $0.99(0.56-1.85)$ & 0.98 \\
\hline Retired & $1460(10.4)$ & $2.33(1.57)$ & & $253 / 383$ & $1.84(0.71-4.9)$ & 0.26 & $1.44(0.53-3.90)$ & 0.85 \\
\hline Unemployed & $310(2.2)$ & $2.24(1.59)$ & & $265 / 178$ & $1.81(0.75-4.9)$ & 0.31 & $1.41(0.51-3.82)$ & 0.86 \\
\hline Others & $254(1.8)$ & $2.12(1.51)$ & & $197 / 282$ & $1.38(0.76-4.1)$ & 0.29 & $1.11(0.50-2.46)$ & 0.83 \\
\hline Level of education: & & & & & & & & \\
\hline Illiterate or Primary education & $728(5.2)$ & $1.31(1.23)$ & $<0.001$ & $563 / 895$ & 1 & 0.004 & 1 & 0.26 \\
\hline Secondary education & $6732(48.1)$ & $2.11(1.36)$ & & $2765 / 2950$ & $2.81(1.3-5.71)$ & & $3.61(1.71-7.74)$ & \\
\hline Diploma degree & $3102(22.2)$ & $2.07(1.26)$ & & $1531 / 2063$ & $2.79(1.3-5.83)$ & 0.006 & $4.60(2.11-10.43)$ & 0.21 \\
\hline University & $2520(18.0)$ & $2.07(1.41)$ & & $968 / 813$ & $2.49(1.2-5.42)$ & 0.023 & $4.18(1.79-9.78)$ & 0.08 \\
\hline$\leq 2000(\mathrm{R})$ & $3110(22.2)$ & $1.82(1.39)$ & 0.291 & $1256 / 1807$ & 1 & 0.61 & --- & --- \\
\hline $2001-3000$ & $3392(24.2)$ & $2.10(1.23)$ & & $1341 / 1987$ & $1.17(0.78-1.8)$ & & --- & \\
\hline $3001-4000$ & 2396(17.1) & 2.11(1.39) & & $1019 / 1154$ & $1.1(0.79-1.83)$ & 0.67 & --- & --- \\
\hline $4001-5000$ & $1720(12.3)$ & $2.03(1.21)$ & & $669 / 1110$ & $0.99(0.53-1.8)$ & 0.91 & --- & --- \\
\hline$>5000$ & $3382(24.2)$ & $2.09(1.39)$ & & $1393 / 2264$ & $1.17(0.78-1.9)$ & 0.62 & --- & --- \\
\hline
\end{tabular}

(a) Analysis using Analysis of Variance (ANOVA).

CI confidence interval, OR odds ratio, $\mathrm{R}$ reference group, LE Egyptian pounds, SD standard deviation.

\section{Multivariate Analysis}

Multivariate logistic regression of significant factors which affected participants' answers revealed that knowledge and awareness about HBV infection were affected by age category of participants, their residence and increased level of education, Table 3 and 4.

While for $\mathrm{HCV}$ infection, awareness about $\mathrm{HCV}$ infection was significant with participants' age category, residence and level of education, Table 8. In addition to these factors, knowledge about $\mathrm{HCV}$ infection was affected by the monthly income of these participants, Table 7.

\section{Discussion}

Hepatitis C constitutes a major health problem in Egypt, which has strong negative clinical, social and economic impact on patients and their families and also on the healthcare systems. Many studies tried to measure the level of knowledge and awareness about $\mathrm{HCV}$ and HBV infections among different groups of population in Egypt. But, results of these studies were heterogonous (Shalaby et al., 2010; Norton et al., 2014).

This community-based cross sectional study was conducted on 14000 residents of Menoufia Governorate residents of different socio-economic and education levels in order to provide comprehensive data about knowledge and awareness of community members about $\mathrm{HCV}$ and $\mathrm{HBV}$ infections.

In this study, we found that $81.9 \%$ of participants had good knowledge about HBV infection and $92.13 \%$ had good awareness about HBV prevention. This was surprisingly higher than expected especially when compared with other studies from countries with high prevalence of $\mathrm{HBV}$ infection.

In a study by Rajamoorthy et al. (2019) they found only $36.9 \%$ of their study population had good knowledge and $38.8 \%$ had good awareness about HBV infection. 
In another study on healthcare workers and University students at Malaysia, they also revealed that $39.1 \%$ of participants had good knowledge and $37.2 \%$ had good awareness about HBV infection (Lim and Rashwan, 2003).

On the other hand, participants' knowledge in our study about HCV infection was less than for $\mathrm{HBV}$ infection $(79.3 \%)$. This may be attributed to false concepts about disease curability. This agreed with other studies on public population in Egypt which revealed lack of knowledge about HCV transmission (Chemaitelly et al., 2014; Sultan et al., 2018). But, awareness about HCV prevention was good among participants (92\%).

Recently, many attempts occurred to improve awareness about viral hepatitis in Egypt by the Information, Education and Communication systems through hotlines, counseling, vaccination campaigns and celebration of World Hepatitis Day. The World Hepatitis Day celebration brought stakeholders together and conveyed important messages to the community (Wanis et al., 2014).

In our study, many participants stated that they had their information about HCV and HBV infection through television programs or newspapers, which reflects the success of this policy as regard improved knowledge and awareness about viral hepatitis in our study population.

This agreed with a study by Shalaby et al. (2010) who assumed that friends and relatives $(47.9 \%)$, television (43\%), newspapers $(36.7 \%)$ and doctors $(30 \%)$ were the main sources of information. Also, Chemaitelly et al. (2014) stated that the media is the main sources of HCV knowledge.

The level of education (illiterate, primary or secondary education, diploma, university and postgraduate levels) was one of the most important factors that affected knowledge and awareness about $\mathrm{HCV}$ and $\mathrm{HBV}$ infections. Also, there was difference between medical and non-medical students.

These results were similar to findings reported by the study at the University of Dammam, Kingdom of Saudi Arabia (Chemaitelly et al., 2014), at University of Lome students (Sultan et al., 2018), in the Medical College of Bitola (Wanis et al., 2014) and in medical colleges of Karachi, Pakistan, which revealed excellent knowledge about HBV and HCV transmission (Almansour et al., 2017).

The impact of education on knowledge about HBV infection had been reported also in studies from Australia (Bagny et al., 2015), Canada (Prodanovska Stojcevska et al., 2010; Khan et al., 2010), British Columbia, China (Hajarizadeh et al., 2015), Kenya (Wu et al., 2009), Poland (Yau et al., 2016)], Singapore (Han et al., 2017) and among Cambodian Americans in the US (Ngaira et al., 2016). But in another study at Malaysia among university students, only $50.3 \%$ of the participants had good Knowledge about HBV infection (Ganczak et al., 2016).

In our study, the socioeconomic level of participants (measured by the monthly income and job of participant) affected knowledge but did not affect awareness about $\mathrm{HCV}$ and HBV infections which agreed with other studies (Wai et al., 2005; Taylor et al., 2002; Ahmad et al., 2016; Tosun et al., 2018). This may explain difference between answers of residents or rural than those of urban areas in our study.

In our study, most of the participants were high level students (medical and non-medical) and on the other hand, one half of included public participants were living in urban areas with better socioeconomic and education levels. This may explain the relatively better levels of knowledge and awareness about HCV and $\mathrm{HBV}$ infection.

Another point to be considered is the time of the study between 2018 and 2019, which was parallel to the successful national project of screening and treating $\mathrm{HCV}$ in Egypt. During this period, many campaigns, television programs, newspapers widely discussed the problems of HCV and other viral infections. This may have helped to raise community knowledge and awareness about these two health problems

\section{Conclusion}

Knowledge and awareness about $\mathrm{HBV}$ and $\mathrm{HCV}$ infections is the base at which the solution of these health problems should be built. Despite of the good results of this study about the level of knowledge and awareness about $\mathrm{HBV}$ and HCV infection and prevention, there is a need to do more studies on different population sectors at various socioeconomic and educational levels.

\section{Acknowledgment}

We wish to thank all the patients and medical staff who participated in this study.

\section{Authors' Contributions}

Wesam S Morad: Contributed to study concept, design and data collection and statistical analysis and interpretation of the data, writing of the paper, critically revised and finalized paper and read approved the final manuscript.

Maha Elsabaawy and Mahmoud H Allam: Clinical investigator in the study contributed to writing of the paper and read approved the final manuscript.

\section{Ethics Approval and Consent to Participate}

This study was conducted in accordance with the International Conference on Harmonisation guideline for good clinical practice and the ethical principles of the 
Declaration of Helsinki. All patients gave written informed consent, which was reviewed and approved by an independent ethics committee or institutional review board of National Liver Institute (IRB00003467). This study was approved (approval numbers 00129/2018).

\section{Consent for Publication}

Patients provided written informed consent for use of their anonymized and aggregated data for research and sharing with other parties.

\section{Data Availability Statement}

The data used to support the findings of this study were supplied by National Liver Institute, Menoufia University under license and so cannot be made freely available. Requests for access to these data should be made to [National Liver Institute Top manager, Menoufia Governorate, Egypt].

The qualitative and quantitative data used to support the findings of this study are restricted by the [National Liver Institute ETHICS BOARD] in order to protect [PATIENT PRIVACY]. Data are available from [National Liver Institute Top manager, Menoufia Governorate, Egypt] for researchers who meet the criteria for access to confidential data.

The qualitative and quantitative data used to support the findings of this study are available from the corresponding author upon request for researchers who meet the criteria for access to confidential data.

The qualitative and quantitative data used to support the findings of this study have not been made available because [National Liver Institute Top manager].

\section{Funding Statement}

This paper was funded by the authors of the paper themselves with no funding agency or funded personnel.

\section{References}

Ahmad, A., L.M. Sann and H.A. Rahman, 2016. Factors associated with knowledge, attitude and practice related to hepatitis $\mathrm{B}$ and $\mathrm{C}$ among international students of Universiti Putra Malaysia. BMC Public Health. DOI: 10.1186/s12889-016-3188-5

Almansour, A., M. Darwish and M.A. Wahab, 2017. Hepatitis C infection awareness among fourth year medical students at University of Dammam. J. Fam Community Med., 24: 49-54.

DOI: $10.4103 / 2230-8229.197182$

Armstrong, G.L., M.J. Alter, G.M. McQuillan and H.S. Margolis, 2000. The past incidence of hepatitis C virus infection: Implications for the future burden of chronic liver disease in the United States. Hepatology, 31: 777-782.

DOI: $10.1002 /$ hep.510310332
Bagny, A., O. Bouglouga, M.A. Djibril, Y.L. Kaaga and A. Dusabe et al., 2015. Knowledge and attitudes of students towards viral hepatitis B and C at the University of Lome. Indian J. Gastroenterol., 34: 78-79. DOI: 10.1007/s12664-014-0479-6

Bruggmann, P., T. Berg, A.L. Øvrehus, C. Moreno and C.E. Brandao Mello et al., 2014. Historical epidemiology of Hepatitis C Virus (HCV) in selected countries. J. Viral Hepat., 21: 5-33.

Chemaitelly, H., L.J. Abu-Raddad and F.D. Miller, 2014. An apparent lack of epidemiologic association between hepatitis $\mathrm{C}$ virus knowledge and the prevalence of hepatitis $\mathrm{C}$ infection in a national survey in Egypt. PLoS One, 8: e69803-e69803. DOI: 10.1371/journal.pone.0069803

Denniston, M.M., R.M. Klevens, G.M. McQuillan and R.B. Jiles, 2012. Awareness of infection, knowledge of hepatitis $\mathrm{C}$ and medical follow-up among individuals testing positive for hepatitis C: National Health and nutrition examination survey 2001-2008. Hepatology, 55: 1652-1661. DOI: $10.1002 /$ hep. 25556

Doss, W., M.K. Mohamed, G. Esmat, M. El Sayed and A. Fontanet et al., 2008. Egyptian national control strategy for viral hepatitis 2008-2012. Arab Republic of Egypt, Ministry of Health and Population, National Committee for the Control of Viral Hepatitis.

Du, J., Z. Wang, B. Xie and M. Zhao, 2012. Hepatitis C knowledge and alcohol consumption among patients receiving methadone maintenance treatment in shanghai, China. Am. J. Drug Alcohol Abuse, 38: 228-232. DOI: 10.3109/00952990.2011.643974

EESJU, 2014. HCV treatment in Egypt. Why cost remains a challenge? Egyptian Economic and Social Justice Unit.

El-Akel, W., M.H. El-Sayed, M. El Kassas, M. El-Serafy and M. Khairy et al., 2017. National treatment programme of hepatitis C in Egypt: Hepatitis C virus model of care. J. Viral Hepat., 24: 262-267. DOI: $10.1111 /$ jvh. 12668

EMHP, 2017. Plan of action for the prevention, care and treatment of viral hepatitis, Egypt 2014-2018. Egyptian Ministry of Health and Population.

Estes, C., M. Abdel-Kareem, W. Abdel-Razek, E. Abdel-Sameea and M. Abuzeid et al., 2015. Economic burden of hepatitis $\mathrm{C}$ in Egypt: The future impact of highly effective therapies. Aliment Pharmacol. Ther., 42: 696-706. DOI: 10.1111/apt.13316

Gamal, E., 2014. Situation of HCV in Egypt: Towards an End to HCV Epidemic.

Ganczak, M., G. Dmytrzyk-Danilow, M. Korzen, M. Drozd-Dabrowska and Z. Szych, 2016. Prevalence of $\mathrm{HBV}$ infection and knowledge of hepatitis B among patients attending primary care clinics in Poland. J. Commun. Health, 41: 635-644. DOI: $10.1007 / \mathrm{s} 10900-015-0139-5$ 
Hajarizadeh, B., J. Wallace, J. Richmond, N. Ngo and C. Enright, 2015. Hepatitis B knowledge and associated factors among people with chronic hepatitis B. Aust. Nz. J. Publ. Heal., 39: 563-568. DOI: $10.1111 / 1753-6405.12378$

Han, Z., Y. Yin, Y. Zhang, S. Ehrhardt and C.L. Thio et al., 2017. Knowledge of and attitudes towards hepatitis B and its transmission from mother to child among pregnant women in Guangdong Province, China. PLoS One, 12: e0178671-e0178671. DOI: 10.1371/journal.pone.0178671

Ismail, S.A., D.F. Cuadros and L. Benova, 2017. Hepatitis B in Egypt: A cross-sectional analysis of prevalence and risk factors for active infection from a nationwide survey. Liver Int., 37: 1814-1822. DOI: 10.1111/liv.13469

Khan, N., S.M. Ahmed, M.M. Khalid, S.H. Siddiqui and A.A. Merchant, 2010. Effect of gender and age on the knowledge, attitude and practice regarding hepatitis $\mathrm{B}$ and $\mathrm{C}$ and vaccination status of hepatitis B among medical students of Karachi, Pakistan. J. Pak. Med. Assoc., 60: 450-455.

Kim, D.D., D.W. Hutton, A.A. Raouf, M. Salama and A. Hablas et al., 2015. Cost-effectiveness model for hepatitis $\mathrm{C}$ screening and treatment: Implications for Egypt and other countries with high prevalence. Global Public Health, 10: 296-317.

Lavanchy, D., 2011. Evolving epidemiology of hepatitis C virus. Clin. Microbiol. Infect., 17: 107-115. DOI: 10.1111/j.1469-0691.2010.03432.x

Liakina, V., S. Hamid, J. Tanaka, S. Olaff Son and A.I. Sharara et al., 2015. Historical epidemiology of Hepatitis C Virus (HCV) in select countries volume 3. J. Viral Hepat., 4: 4-20.

Lim, H. and H. Rashwan, 2003. Awareness of hepatitis A and hepatitis B among residents in Kuala Lumpur and Selangor. Malaysian J. Pharm., 1: 76-85.

McNeil Jr., D.G., 2015. Curing Hepatitis C, in an experiment the size of Egypt. The New York Times.

MHPICFI, 2015. Egypt health issues survey 2015. Cairo, Egypt and Rockville, Ministry of Health and Population and ICF International, Maryland, USA.

Mohd Hanafiah, K., J. Groeger, A.D. Flaxman and S.T. Wiersma, 2013. Global epidemiology of hepatitis C virus infection: New estimates of age-specific antibody to HCV seroprevalence. Hepatology, 57: 1333-1342. DOI: 10.1002/hep.26141

Ngaira, J.A., J. Kimotho, I. Mirigi, S. Osman and Z. Ng'ang'a et al., 2016. Prevalence, awareness and risk factors associated with hepatitis B infection among pregnant women attending the antenatal clinic at Mbagathi District Hospital in Nairobi, Kenya. Pan Afr. Med. J., 24: 315-315.

DOI: 10.11604/pamj.2016.24.315.9255
Norton, B.L., C.I. Voils, S.H. Timberlake, E.J. Hecker and N.D. Goswami et al., 2014. Community-based HCV screening: Knowledge and attitudes in a high risk urban population. BMC Infect. Dis., 14: 1-9. DOI: 10.1186/1471-2334-14-74

Pépin, J., C.N. Abou Chakra, E. Pépin, V. Nault and L. Valiquette, 2014. Evolution of the global burden of viral infections from unsafe medical injections, 2000-2010. PLoS One, 9: e99677-e99677.

DOI: 10.1371/journal.pone.0099677

Prodanovska Stojcevska, V., R. Isjanovska and E. Popova Ramova, 2010. Knowledge of HCV infection among nursing students of the Medical College of Bitola. Arh. Hig. Rada Toksikol., 61: 197-201. DOI: 10.2478/10004-1254-61-2010-1990

Rajamoorthy, Y., N.M. Taib, S. Munusamy, S. Anwar, S. Anwar and A.L. Wagner et al., 2019. Knowledge and awareness of hepatitis B among households in Malaysia: a community-based cross-sectional survey. Public Health, 19: 47-47.

DOI: $10.1186 / \mathrm{s} 12889-018-6375-8$

Saraswat, V., S. Norris, R.J. de Knegt, J.F. Sanchez Avila and M. Sonderup et al., 2015. Historical epidemiology of Hepatitis C Virus (HCV) in select countries - volume 2. J. Viral Hepat., 1: 6-25.

Shalaby, S., I.A. Kabbash, G. El Saleet, N. Mansour and A. Omar et al., 2010. Hepatitis B and $\mathrm{C}$ viral infection: Prevalence, knowledge, attitude and practice among barbers and clients in Gharbia governorate, Egypt. East Mediterr Health J., 16: 10-17. DOI: $10.26719 / 2010.16 .1 .10$

Stanaway, J.D., A.D. Flaxman, M. Naghavi, C. Fitzmaurice and T. Vos et al., 2016. The global burden of viral hepatitis from 1990 to 2013: findings from the Global Burden of Disease Study, 2013. Lancet, 388: 1081-1088. DOI: 10.1016/S0140-6736(16)30579-7

Sultan, N.Y., A. YacoobMayet, S.A. Alaqeel and H.A. Al-Omar, 2018. Assessing the level of knowledge and available sources of information about hepatitis $\mathrm{C}$ infection among $\mathrm{HCV}$-infected Egyptians. BMC Public Health, 18: 747-747.

DOI: $10.1186 / \mathrm{s} 12889-018-5672-6$

Taylor, V.M., J.C. Jackson, N. Chan, A. Kuniyuki and Y. Yasui, 2002. Hepatitis B knowledge and practices among Cambodian American women in Seattle, Washington. J. Commun. Health, 27: 151-163. DOI: 10.1023/A:1015229405765

Tosun, S., O. Aygün, H.Ö. Özdemir, E. Korkmaz and D. Özdemi, 2018. The impact of economic and social factors on the prevalence of hepatitis B in Turkey. BMC Public Health, 18: 649-649. DOI: $10.1186 / \mathrm{s} 12889-018-5575-6$ 
Toy, M., F.O. Önder, T. Wörmann, A.M. Bozdayi and S.W. Schalm et al., 2011. Age- and region-specific hepatitis B prevalence in Turkey estimated using generalized linear mixed models: A systematic review. BMC Infect. Dis., 11: 337-337.

DOI: $10.1186 / 1471-2334-11-337$

Wai, C.T., B. Mak, W. Chua, M.H. Tan and S. Ng et al., 2005. Misperceptions among patients with chronic hepatitis B in Singapore. World J. Gastroentero, 11: 5002-5005. DOI: 10.3748/wjg.v11.i32.5002

Wanis, H., A. Hussein and A. El Shibiny, 2014. HCV treatment in Egypt - why cost remains a challenge Egyptian initiative for personal rights.

WHO, 2012. Prevention and control of viral hepatitis infection. Framework for Global Action. WHO/HSE/PED/HIP/GHP.

WHO, 2016. Global report on access to hepatitis C treatment: Focus on overcoming barriers. World Health Organization, Geneva.
WHOEB, 2009. World Health Organization Executive Board. Viral hepatitis. Report by the Secretariat.

Williams, I.T., B.P. Bell, W. Kuhnert and M.J. Alter, 2011. Incidence and transmission patterns of acute hepatitis C in the United States, 1982-2006. Arch Intern Med., 171: 242-248.

DOI: 10.1001/archinternmed.2010.511

Wu, H., C. Yim, A. Chan, M. Ho and J. Heathcote, 2009. Sociocultural factors that potentially affect the institution of prevention and treatment strategies for hepatitis $\mathrm{B}$ in Chinese Canadians. Can. J. Gastroenterol., 23: 31-36.

DOI: $10.1155 / 2009 / 608352$

Yau, A.H., J.A. Ford, P.W. Kwan, J. Chan and Q. Choo et al., 2016. Hepatitis B awareness and knowledge in Asian communities in British Columbia. Can. J. Gastroenterol. Hepatol., 2016: 4278724-4278724. DOI: $10.1155 / 2016 / 4278724$ 